\title{
Guest editorial introduction to the special issue on biomedical microneedles
}

\author{
Boris Stoeber ${ }^{1} \cdot$ Mikolaj Milewski $^{2}$ \\ Accepted: 26 November 2020 / Published online: 14 January 2021 \\ (C) The Author(s), under exclusive licence to Springer Science+Business Media, LLC part of Springer Nature 2021
}

\section{Introduction}

The human skin consists of several layers; the outermost layer, the stratum corneum, provides mechanical protection from the environment. The underlying viable epidermis and the dermis include a large number of immune cells and rich vascular network. Biomedical microneedles are small mechanical structures that allow penetrating the human skin; in particular, they allow piercing the stratum corneum to reach the viable epidermis and the dermis, either to deliver therapeutics, vaccines and cosmetics or for sensing applications and for stimulation.

Micromachined solid electrode spike arrays have first been introduced in the 1980s, initially intended for measuring neural signals. The 1990s saw the emergence of hollow microneedles, followed by dissolving microneedles in the 2000s. While always a focus of microneedle research, the 2010s have seen a more significant emphasis on sensing applications for microneedles.

This Special Issue was initiated for dissemination of contributions presented at the 5th International Conference on Microneedles, held from May 29 until June 1, 2018 in Vancouver, Canada, while other contributions have also been added to this collection.

This article is part of the Topical collection on Biomedical MicroNeedles

Boris Stoeber

boris.stoeber@ubc.ca

1 Department of Mechanical Engineering, Department of Electrical and Computer Engineering, The University of British Columbia, 2054-6250 Applied Science Lane, Vancouver V6T 1Z4, Canada

2 Pharmaceutical Sciences, Merck \& Co., Inc., Kenilworth, NJ, USA

\section{Recent highlights of this special issue}

This SI consists of 15 articles, grouped in the four categories:

a) Design and manufacturing,

b) Skin mechanics and toxicology,

c) Drug and cell delivery, and

d) Sensing and stimulation.

The category design and manufacturing includes two articles related to the release of compounds from a dissolvable matrix. One focuses on the formulation of the dissolvable material that is then used to fabricate dissolvable needles. The other article discusses microneedle surface structures that improve coating with a dissolvable material. Additionally, another report assesses the design and fabrication of hafnium oxide microneedles.

Pawar and Shende report on the development and testing of dissolvable microneedles for the intradermal delivery of artemether and lumefantrine. They optimize the manufacturing of lumefantrine nanoparticles and encapsulate these nanoparticles together with chitosan in a matrix of a blend of biocompatible and dissolvable polymers that form the microneedles. They demonstrate in vitro and ex-vivo release of the two compounds within $24 \mathrm{~h}$.

Plamadeala et al. take a boimimetics approach to the design of microneedles that have a surface inspired by European true bugs. The surface features enhance the rapid coating of the microneedles with drugs and vaccines. The authors create these needles using two-photon polymerization and demonstrate replication through micromolding. They compare the surface coating of microneedles with and without the surface features, and they perform ex-vivo and in-vivo tests.

Zhang et al. demonstrate a method of hafnium oxide hollow microneedle fabrication based on deep reactive ion etching of silicon and atomic layer deposition of hafnium oxide. 
Additionally, the authors present a finite element analysis of the microneedles as applied to different designs of microneedle arrays and the 'bed of nail effect' on the skin.

There are three articles in the category skin mechanics and toxicology. One focuses on the parameters involved with dynamic insertion of microneedles into the skin. Another article investigates the formation of large pores in the skin by inserting microneedles and how these pores enhance the delivery of compounds into the skin. The third article includes a literature review on the toxicology of microneedles related to their insertion into human skin.

Ranamukhaarachchi and Stoeber investigate the process of dynamic microneedle insertion into skin to evaluate the impact of the different insertion parameters. They use an artificial human skin model and find that the microneedle velocity at impact is the most critical parameter for successful insertion. They determine correlations between the microneedle tip area or the number of needles in an array and the insertion force.

Kim et al. set out to investigate in vitro delivery of magnesium ascorbyl phosphate (MAP) across porcine ear skin pretreated with microneedles made out of hyaluronic acid. First, micromolding technique is used to fabricate hyaluronic acid microneedles with MAP. Then effective piercing of the skin is demonstrated with dye binding and histology confirmed formation of microchannels. Finally, in vitro Franz diffusion experiments are carried out showing around 2-fold improvement in MAP flux across skin when skin was pretreated with microneedles.

Cary et al. examine the safety aspects of dermal microneedles usage. Several risk factors including infection, contact dermatitis, chronic inflammatory reactions and granulomas, scarring and post-inflammatory hyperpigmentation cases are described in detail. Authors conclude that the use of microneedles in the cosmetic industry for rejuvenation purposes raises some safety concerns related to improper sterilization and inappropriate coupling of topical products when carried out in an out-patient setting.

In the category of drug and cell delivery four reports highlight recent advances in the field. One study is based on microneedle-assisted in vitro permeation and quantification of therapeutic agents in ex vivo tissue while two report in vivo delivery of therapeutic agents either topically or systemically. Parameters such as microneedle skin insertion, kinetics of drug transfer, and delivery into skin or systemic circulation are described. Another study reports on microneedle-based cell delivery for cellular therapies.

Kelchen and Brogden address the topic of topical betablocker formulation use to treat infantile hemangiomas. Specifically, authors investigate in vitro local skin tissue concentration and permeation across skin of two common therapeutics, propranolol and timolol, in presence of a microneedle pretreatment. Different physicochemical properties of these two permeants are evoked to rationalize different in vitro behavior.

Nguyen et al. study a microneedle-based topical treatment of cutaneous leishmaniasis with amphotericin B. In vivo studies include different lengths of microneedles $(500 \mu \mathrm{m}-$ $1000 \mu \mathrm{m})$ in mice infected by either Leishmania mexicana or Leishmania major. Authors find that new treatment options show promise treating small nodules caused by Leishmania mexicana but are of limited effectiveness against a disseminated Leishmania major infection.

Kapoor et al. investigate the suitability of a coated solid microstructure transdermal system (sMTS) to promote chemical stability and facilitate systemic delivery of a therapeutic peptide in Yucatan minipigs. Authors demonstrate improved stability characteristics as compared to a liquid-based subcutaneous injection and demonstrate a high-efficiency in vivo transfer of peptide from microneedle structures into skin. Also, pharmacokinetics of intradermal and subcutaneous delivery are discussed.

Chen et al. deliver cells into a model tissue with the application to cellular therapies. They seed cells on the surface of a microneedle array. A high percentage of these cells is successfully delivered into the target location of collagen hydrogel that served as model tissue. The authors demonstrate cell delivery for $\mathrm{HaCaT}$ cells, a spontaneously immortalized human keratinocyte cell line, and for human follicle dermal papilla cells.

The category sensing and stimulation includes fife articles. Four articles focus on the interstitial fluid of the skin to measure biomarkers. Two of these articles demonstrate porous microneedle arrays for the extraction of this fluid from the skin; another article uses solid microneedles to pierce the skin followed by interstitial fluid collection with filter paper; the other article integrates a biosensor into the lumen of a microneedle for sensing while the microneedle is inserted into skin. The fifth article uses micro electrode arrays for electric nerve stimulation.

Chen et al. demonstrate a device for extracting interstitial fluid from the skin for the subsequent offline analysis of biomarkers. They develop a process for fabricating sponge-like microneedle patches from polymer that rapidly absorb interstitial fluid from the skin once inserted. The fluid is then removed for analysis through centrifugation.

Takeuchi et al. present a microfluidic chip for the extraction of interstitial fluid and on-chip analysis. They also use an array of porous microneedles; however, they integrate flow channels into the chip underneath the needles for the transport of the interstitial fluid once extracted. The fluid is directed to an analysis area on the chip through an integrated capillary pump.

Kolluru et al. collect interstitial fluid from a rat into filter paper attached to a microneedle device after perforating the rat skin several times with the microneedles. They demonstrate a 
comparable vancomycin concentration in the extracted interstitial fluid as in serum. In addition, the authors measure a similar antibody concentration in the interstitial fluid as in plasma.

Ribet et al. develop a miniaturized continuous glucose monitor based on a single microneedle. They micromachine a small electrochemical glucose sensor that they insert into the lumen of a microneedle. The authors demonstrate continuous sensing of glucose in the interstitial fluid of the skin of a human forearm; the sensor signal follows changes in capillary blood from reference measurements.

Soltanzadeh et al. describe fabrication of skin surface microneedle electrodes for electrical peripheral nerve stimulation. The authors then test performance of new electrodes as compared with conventional surface electrodes in nerve conduction velocity assay and demonstrate that the electrode-skin contact resistance decreases and interface capacitance and charge transfer resistance are improved with newly designed electrodes. Also, 3-dimensional finite element simulations are carried out to study current density in the deep layers of the skin.

\section{Future trends in biomedical microneedles}

The microneedle field has evolved substantially over the last decade. The number of scientific reports related to microneedle array design and manufacture, skin response to external stimuli, drug and vaccine delivery, and micro-sensory applications had been growing steadily adding to the body of knowledge and experience. However, it is perhaps the microneedle application aspect which gained substantially more traction recently and holds the most promise for the field by introducing it into the canons of vaccine and drug delivery options. Examples such as a publication by E. Kim, et al. "Microneedle Array Delivered Recombinant Coronavirus Vaccines: Immunogenicity and Rapid Translational Development" where authors designed microneedle arrays containing MERS-CoV-S1 and SARS-CoV-2 vaccines generating potent antigen-specific IgG responses in mice show the timeliness, flexibility, and maturation of this field in addressing most pressing health challenges of our times. We expect that vaccine development will continue to be at the forefront of the biomedical microneedle field. Additionally, microneedle-assisted macromolecule delivery not limited to skin but also encompassing ocular or buccal tissues are likely to grow in significance. The next major milestone for this research field would be a more widespread bench-to-bedside translation from prototype concepts into vaccine and drug products.

Acknowledgments We thank all the contributing authors for the submission of their latest research results on biomedical microneedles to this Special Issue. We are also thankful for all the reviewers and their time and invaluable comments that allowed us to complete this Special Issue. Finally, we thank Prof. Mauro Ferrari for the exciting opportunity to organize this Special Issue on biomedical microneedles.

Publisher's note Springer Nature remains neutral with regard to jurisdictional claims in published maps and institutional affiliations. 